\title{
Dynamic optic nerve sheath diameter responses to short-term hyperventilation measured with sonography in patients under general anesthesia
}

\author{
Ji-Yeon Kim, Hong-Gi Min, Seung-Il Ha, Hye-Won Jeong, Hyungseok Seo, and Joung-Uk Kim \\ Department of Anesthesiology and Pain Medicine, Asan Medical Center, University of Ulsan College of Medicine, Seoul, Korea
}

Background: Rapid evaluation and management of intracranial pressure (ICP) can help to early detection of increased ICP and improve postoperative outcomes in neurocritically-ill patients. Sonographic measurement of optic nerve sheath diameter (ONSD) is a non-invasive method of evaluating increased intracranial pressure at the bedside. In the present study, we hypothesized that sonographic ONSD, as a surrogate of ICP change, can be dynamically changed in response to carbon dioxide change using short-term hyperventilation.

Methods: Fourteen patients were enrolled. During general anesthesia, end-tidal carbon dioxide concentration $\left(\mathrm{ETCO}_{2}\right)$ was decreased from $40 \mathrm{mmHg}$ to $30 \mathrm{mmHg}$ within 10 minutes. ONSD, which was monitored continuously in the single sonographic plane, was repeatedly measured at 1 and 5 minutes with $\mathrm{ETCO}_{2} 40 \mathrm{mmHg}$ (time-point 1 and 2) and measured again at 1 and 5 minutes with $\mathrm{ETCO}_{2} 30 \mathrm{mmHg}$ (time-point 3 and 4).

Results: The mean \pm standard deviation of ONSD sequentially measured at four time-points were $5.0 \pm 0.5,5.0 \pm 0.4$, $3.8 \pm 0.6$, and $4.0 \pm 0.4 \mathrm{~mm}$, respectively. ONSD was significantly decreased at time-point 3 and 4, compared with 1 and $2(\mathrm{P}<0.001)$.

Conclusions: The ONSD was rapidly changed in response to $\mathrm{ETCO}_{2}$. This finding may support that ONSD may be beneficial to close ICP monitoring in response to $\mathrm{CO}_{2}$ change. (Korean J Anesthesiol 2014; 67: 240-245)

Key Words: Carbon dioxide, Hyperventilation, Optic nerve sheath diameter, Ultrasonography.

Received: May 15, 2014. Revised: 1st, June 18, 2014; 2nd, June 23, 2014. Accepted: June 25, 2014.

Corresponding author: Hyungseok Seo, M.D., Department of Anesthesiology and Pain Medicine, Asan Medical Center, University of Ulsan College of Medicine, 88, Olympic-ro 43-gil, Songpa-gu, Seoul 138-736, Korea. Tel: 82-2-3010-5683, Fax: 82-2-470-1363, E-mail: seohyungseok@gmail.com (c) This is an open-access article distributed under the terms of the Creative Commons Attribution Non-Commercial License (http:// creativecommons.org/licenses/by-nc/3.0/), which permits unrestricted non-commercial use, distribution, and reproduction in any medium, provided the original work is properly cited. 


\section{Introduction}

Cerebral hemodynamics, including cerebral blood flow (CBF) and intracranial pressure (ICP) can be affected by many factors such as brain tumor [1] and volatile agents [2]. Carbon dioxide $\left(\mathrm{CO}_{2}\right)$, a potent cerebral vasodilator, is associated with $\mathrm{CBF}$, which may contribute to ICP change [2,3]. It has been reported that the CBF can be affected dynamically, within a few minutes, in response to arterial carbon dioxide concentration $\left(\mathrm{PaCO}_{2}\right)$ change and tight control of $\mathrm{PaCO}_{2}$ can be associated with neurological outcomes [4], as well as important to improve surgical outcomes even in non-neurosurgical patients [5,6]. Therefore, $\mathrm{PaCO}_{2}$ should be deliberately monitored during mechanically ventilated patients, particularly in neurocritically-ill patients. However, $\mathrm{PaCO}_{2}$ can be measured by arterial blood gas analysis, thereby being inappropriate for continuous monitoring and rapid assessment of systemic $\mathrm{CO}_{2}$ level change. The end-tidal carbon dioxide concentration $\left(\mathrm{ETCO}_{2}\right)$ has been reported to be a non-invasive parameter to estimate $\mathrm{PaCO}_{2}$ indirectly [7], and is routinely monitored during general anesthesia.

Rapid evaluation and management of ICP can be helpful in early detection of increased ICP and improve postoperative outcomes in neurocritically-ill patients $[8,9]$. Although direct methods of measuring ICP are considered the gold standard, these methods carry risks, including bleeding and infection [10]. Ultrasonography of optic nerve sheath diameter (ONSD) is a non-invasive, indirect technique of evaluating changes in ICP and it shows a good correlation with direct ICP measurement. A previous study showed that ONSD is linearly correlated with ICP and ONSD > $5.7 \mathrm{~mm}$ suggested of ICP increase $>20 \mathrm{mmHg}$ [11]. Although ONSD is well responsive to ICP change [12], it may show a delayed response in the case of highly increased ICP $[12,13]$. Moreover, despite of the association between ONSD and $\mathrm{PaCO}_{2}$ [14], the dynamic responsiveness of ONSD, in association with acute arterial $\mathrm{CO}_{2}$ change which can affect ICP, still has not been well investigated.

In the present study, we hypothesized that the sonographic ONSD, as a surrogate for the ICP, can rapidly change in response to arterial $\mathrm{CO}_{2}$ change by monitoring $\mathrm{ETCO}_{2}$ reduction using short-term hyperventilation. To investigate the rapid change of ONSD, we monitor $\mathrm{ETCO}_{2}$, instead of $\mathrm{PaCO}_{2}$, to adjust systemic $\mathrm{CO}_{2}$ level constantly throughout the research. For the evaluation of acute response of ONSD, we measured ONSD in a continuous fashion on a single fixed transverse sonographic plane.

\section{Materials and Methods}

\section{Patients}

Fourteen adult patients admitted for elective anterior cervi- cal discectomy fusion surgery from December 2012 to March 2013 were enrolled. Patients suspected of having increased ICP were excluded with the criteria of less than 20 years of age, with known orbital disorder, or with history of previous intracranial surgery. All patients enrolled were provided written informed consent. The study protocol was approved by the Institutional Review Board of the hospital and was registered with the Clinical Research Informational Service (KCT 0000573, http://cris. nih.go.kr).

\section{Anesthetic protocols}

None of the patients was prescribed with premedication. General anesthesia was induced and maintained by targetcontrolled infusions of propofol and remifentanil via an infusion pump (TCI pump, Orchestra Base Primea, Fresenius Vial, France). The depth of anesthesia was equally maintained at a bispectral index (BIS) between 40 and 60, as assessed with a BIS A-1050 Monitor (Aspect Medical Systems, Newton, MA, USA). Rocuronium bromide $0.7 \mathrm{mg} / \mathrm{kg}$ was used to facilitate tracheal intubation. Direct arterial pressure was monitored by radial arterial catheterization. The ambient operating room temperature was kept at $25-26^{\circ} \mathrm{C}$.

Fresh gas flow was maintained at $3 \mathrm{~L} / \mathrm{min}$ of medical air containing 50\% oxygen. Mechanical ventilation was applied using a tidal volume of $8 \mathrm{ml} / \mathrm{kg}$, with a respiratory rate of $8-10$ breaths/ min to maintain normocapnia during surgery. After induction, an ultrasound probe (L25x/M-turbo, Sonosite Inc., Bothell, WA, USA) was placed onto the patient's right upper eyelid and was fixed manually to maintain a consistent sonographic view. To adjust $\mathrm{ETCO}_{2}$ close to $40 \mathrm{mmHg}$ and $30 \mathrm{mmHg}$, the respiratory rate was changed temporarily from 8-18 breaths/min with a fixed tidal volume. ONSD was measured at four specific timepoints during the mechanical ventilation, at 1 minute after $\mathrm{ETCO}_{2}$ reached $40 \mathrm{mmHg}$ (time-point 1), at 5 minutes after $\mathrm{ETCO}_{2}$ reached $40 \mathrm{mmHg}$ (time-point 2), at 1 minute after $\mathrm{ETCO}_{2}$ reached $30 \mathrm{mmHg}$ (time-point 3), and at 5 minute after $\mathrm{ETCO}_{2}$ reached $30 \mathrm{mmHg}$ (time-point 4). With $\mathrm{ETCO}_{2}$ reaching $40 \mathrm{mmHg}$, this level was maintained for 5 minutes for stabilizing. We measured ONSD at 1 minute and 5 minutes maintaining $\mathrm{ETCO}_{2}$ at $40 \mathrm{mmHg}$ (time-point 1 and 2, respectively). Thereafter, controlled hyperventilation was gradually induced over 10 minutes until the $\mathrm{ETCO}_{2}$ was reduced to $30 \mathrm{mmHg}$ by increasing the one respiratory rate every minute. This level was maintained again for 5 minutes and ONSD was measured at the same time-points as with $\mathrm{ETCO}_{2} 30 \mathrm{mmHg}$ (time-point 3 and 4, respectively). Mean arterial pressure (MAP), heart rate, body temperature, and $\mathrm{PaCO}_{2}$ were measured and the values were averaged in accordance with each $\mathrm{ETCO}_{2}$ maintaining period. Body temperature was measured using an esophageal tempera- 
ture probe (DeRoyal Inc., Powell, TN, USA) and a forced airwarming system (Bair Hugger, Augustine Medical Inc, MN, USA) was used to maintain esophageal temperature at 35.5$37.0^{\circ} \mathrm{C}$.

\section{Sonographic measurement of ONSD}

Two observers who have experiences of more than 40 cases of ONSD measurements were participated in the present study. ONSD was measured using a $7.5 \mathrm{MHz}$ linear probe with orbital imaging settings of the ultrasound machine. All patients were placed in the supine position after induction of general anesthesia. After $\mathrm{ETCO}_{2}$ was reached at $40 \mathrm{mmHg}$ and stabilized, a thick gel layer was applied to each patient's right upper eyelid, and the ultrasound probe was placed on the superior and lateral aspects of the orbit and angled slightly to view the linear hypoechoic structure with clear margins posterior to the globe in the transverse plane. Once the optic nerve sheath was identified, ONSD was measured $3 \mathrm{~mm}$ behind the optic disc and the images were saved. Each ONSD was measured by one independent observer three times and the average value was recorded. During controlled hyperventilation, the observer held the ultrasound probe with the support of a flexible clamp continuously to maintain a consistent sonographic view. Again once $\mathrm{ETCO}_{2}$ reached $30 \mathrm{mmHg}$, ONSD was measured likewise. To assess the reliability between the two observers, all sonographic images were saved and transferred to picture archiving and communication system (PACS) of our institute. Then the other observer would measure the transferred image on PACS through conversion of numbers of pixels in to the scales in length.

\section{Statistical analysis}

Our pilot study showed that the mean difference and standard deviation (SD) of ONSD change were 0.6 and 0.4 , respectively. A type I error of 0.05 and a power of 0.9 indicated that fourteen patients were required for samples. Statistical analysis was performed using SPSS 12.0 (SPSS Inc., Chicago, IL, USA). The Shapiro-Wilk test was used to check the normality of demographic and outcome data. The average ONSD value measured by two independent observers was used for data analysis. Oneway repeated measures analysis of variance was used to compare ONSD values at 4 time-points and Turkey method was used for post-hoc analysis. For assessment of the intraobserver reliability, Cronbach's Alpha, which is a model of internal consistency, was calculated. In order to assess the agreement between the values of the two observers, Bland-Altman analysis was used. Data of MAP, heart rate, body temperature, and $\mathrm{PaCO}_{2}$ were expressed as mean $\pm \mathrm{SD}$. A P value of $<0.05$ was considered statistically significant.

\section{Results}

The demographic and intraoperative data of the 14 patients are shown in Table 1. There were no significant differences among MAP, heart rate, and body temperature at two $\mathrm{ETCO}_{2}$ level ( $\mathrm{ETCO}_{2} 40$ and $\left.30 \mathrm{mmHg}\right)$.

The sequential ultrasound images, which show the consistency of the sonographic plane, were obtained at four different time-points (Fig. 1). The mean \pm SD of ONSD measured at four time-points were $5.0 \pm 0.5,5.0 \pm 0.4,3.8 \pm 0.6$, and $4.0 \pm 0.4 \mathrm{~mm}$ (at time-point 1, 2, 3, and 4, respectively). Fig. 2 showed that ONSD was not significantly different between time-point 1 and 2 , as well as time-point 3 and 4. However, ONSD decreased significantly at the time-point 3 and 4 compared with 1 and $2(\mathrm{P}<$ 0.001). ONSD was changed significantly with change of $\mathrm{ETCO}_{2}$ and was stabilized with the maintenance of $\mathrm{ETCO}_{2}$ level.

The measured values show good intra-observer reliability, as well as acceptable inter-observer variability. Cronbach's Alpha for evaluating intra-observer reliability was 0.93 for observer 1 and 0.94 for observer 2. Bland-Altman analysis showed that the mean difference of the values obtained between the two observers was $0.2 \mathrm{~mm}$, when the limits of agreement (mean \pm 1.96 times of SD) were -0.47 and $0.87 \mathrm{~mm}$.

\section{Discussion}

The major finding of this study is that ONSD, which was measured continuously in a single transverse sonographic plane, showed rapid response to $\mathrm{ETCO}_{2}$ change.

Because the brain is enclosed by the skull, increase in ICP can impede cerebral blood flow and may cause secondary ischemic brain injury. Increase in intra-cerebral volume is initially

Table 1. Demographic Data and Variables at Each ONSD Measurement Demographics

\begin{tabular}{|c|c|c|}
\hline $\begin{array}{l}\text { Age }(\mathrm{yr}) \\
\text { Sex }(\mathrm{M} / \mathrm{F}) \\
\text { Height }(\mathrm{cm}) \\
\text { Weight }(\mathrm{kg})\end{array}$ & \multicolumn{2}{|c|}{$\begin{array}{c}51.1 \pm 8.7 \\
9 / 5 \\
155.6 \pm 29.2 \\
66.7 \pm 8.0\end{array}$} \\
\hline Variables & $\begin{array}{c}\mathrm{ETCO}_{2} \\
40 \mathrm{mmHg}\end{array}$ & $\begin{array}{c}\mathrm{ETCO}_{2} \\
30 \mathrm{mmHg}\end{array}$ \\
\hline MAP (mmHg) & $64.4 \pm 6.2$ & $66.4 \pm 4.7$ \\
\hline HR (beats/min) & $62.5 \pm 14.2$ & $61.4 \pm 13.2$ \\
\hline Body temperature $\left({ }^{\circ} \mathrm{C}\right)$ & $36.3 \pm 0.3$ & $36.1 \pm 0.4$ \\
\hline $\mathrm{PaCO}_{2}(\mathrm{mmHg})$ & $44.8 \pm 3.1$ & $35.4 \pm 2.9^{*}$ \\
\hline $\mathrm{ETCO}_{2}(\mathrm{mmHg})$ & $39.4 \pm 0.9$ & $30.0 \pm 0.6^{*}$ \\
\hline $\mathrm{PaCO}_{2}-\mathrm{ETCO}_{2}(\mathrm{mmHg})$ & $5.4 \pm 3.1$ & $5.6 \pm 2.5$ \\
\hline
\end{tabular}

Data are expressed as mean \pm SD. MAP: mean arterial pressure, HR: heart rate, $\mathrm{PaCO}_{2}$ : arterial partial pressure of carbon dioxide, $\mathrm{ETCO}_{2}$ : end-tidal carbon dioxide concentration, $\mathrm{PaCO}_{2}-\mathrm{ETCO}_{2}$ : the difference between $\mathrm{PaCO}_{2}$ and $\mathrm{ETCO}_{2}$. ${ }^{*} \mathrm{P}<0.001$. 

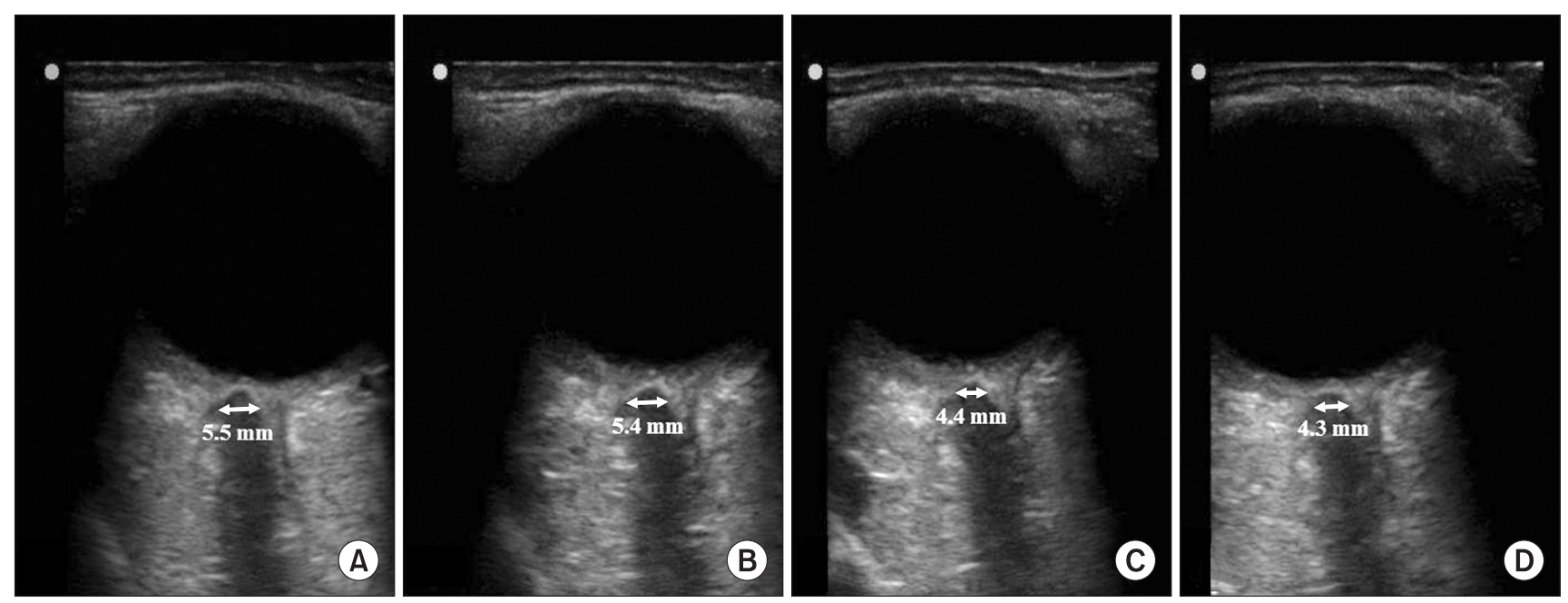

Fig. 1. The sequential ultrasound images at four time-points. (A) ONSD measured at 1 minute after ETCO $\mathrm{E}_{2}$ reached $40 \mathrm{mmHg}$ (time-point 1 ), (B) ONSD measured at 5 minutes after $\mathrm{ETCO}_{2}$ reached $40 \mathrm{mmHg}$ (time-point 2), (C) ONSD measured at 1 minute after $\mathrm{ETCO}_{2}$ reached $30 \mathrm{mmHg}$ (time-point 3), (D) ONSD measured at 5 minutes after $\mathrm{ETCO}_{2}$ reached $30 \mathrm{mmHg}$ (time-point 4). ONSD: optic nerve sheath diameter, ETCO 2 : end-tidal carbon dioxide concentration.

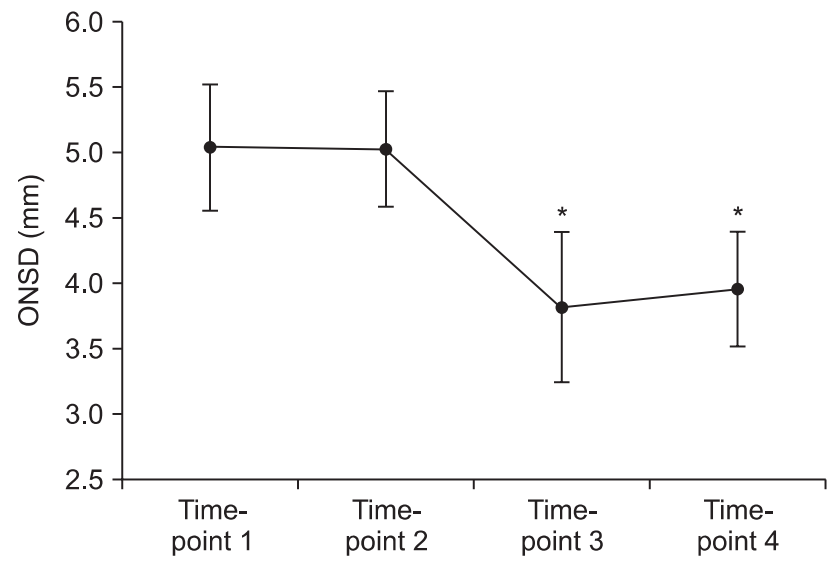

Fig. 2. The change of sonographic ONSD at four specific time-points. ONSD decreased significantly between time-points 2 and $3(\mathrm{P}<0.001)$. ONSD values at time-point 3 and 4 were significantly decreased compared with values at time-point 1 and 2 . ONSD did not show differences between two time-points in the same $\mathrm{ETCO}_{2}$ level (time-point 1 vs. 2, and 3 vs. 4). ONSD: optic nerve sheath diameter, $\mathrm{ETCO}_{2}$ : end-tidal carbon dioxide concentration. ${ }^{*} \mathrm{P}<0.001$ (compared with time-point 1 and 2).

compensated by cerebrospinal fluid extrusion, maintaining a low ICP state. However, exhausted compensatory mechanism results in decrease in brain compliance and rapid increase of ICP associated with small increase of intra-cerebral volume [15]. In patients with traumatic brain injury, increase of ICP is associated with poor outcome and monitoring ICP has been known to decrease mortality $[5,16]$. Moreover, even in non-neurosurgical patients intraoperative ICP monitoring may require for appropriate ICP management [17] for improved neurological outcomes [18].
$\mathrm{CO}_{2}$ is known as a major factor affecting the ICP, even in patients with moderate to severe head injury [19]. ICP monitoring in association with $\mathrm{CO}_{2}$ can be effective in appropriate management of mechanically ventilated patients in intensive care unit, as well as intraoperative period. Short-term hyperventilation can improve autoregulatory response in head injury [20] and even prevent acute ICP increase during reperfusion period of liver transplantation. However, sustained hyperventilation can decrease $\mathrm{CBF}$, resulting in poor prognosis in patients with elevated ICP [19]. Non-invasive monitoring technique such as sonographic ONSD measurement can be promptly applied and performed at bedside to evaluate ICP $[9,21]$ and may help to make optimal strategy in ICP management. Even though patients included in this study did not suffer from intracranial lesion and had normal brain compliance, ONSD showed a significant response in associated $\mathrm{CO}_{2}$ change.

In the present study, same sonographic plane was held still during short-term hyperventilation in order to monitor consistently ONSD. Conventionally, ONSD is determined by averaging the two values obtained from the transverse and sagittal sonographic planes [22]. However, it may require additional time to measure ONSD from two different sonographic views, resulting in interference of the dynamic correlation between $\mathrm{ONSD}$ and $\mathrm{CO}_{2}$ change. Moreover even a subtle movement of ultrasound probe can change the sonographic view and interfere in identifying the accurate boundaries of nerve sheath, causing problems of inconsistency and measurement errors. To maintain consistent sonographic view, the observers held the ultrasound probe with the support of a flexible clamp continuously in the single transverse plane during $\mathrm{ETCO}_{2}$ change. This technique 
may be responsible for high intraobserver reliability of our study and may be useful in minimizing the time-delay in conventional technique, better reflecting the dynamic changes in ONSD. In addition, sonographic measurements of ONSD may vary by the observer's skill [23] or the types of ultrasound devices [24]. To improve the reliability of our measurement technique, all sonographic images were evaluated by two experienced observers independently and the mean difference between the two observers was similar to previous result [22].

We found that ONSD responded rapidly in change to $\mathrm{ETCO}_{2}$, suggesting that ICP change can be occurred within 10 minutes. But, between time-point 1 and 2, as well as 3 and 4, when $\mathrm{ETCO}_{2}$ was maintained for 5 minutes, ONSD did not change. This finding may also suggest that ONSD can reflect the static ICP status at sustained $\mathrm{CO}_{2}$ level, thereby being is useful in monitoring ICP in $\mathrm{CO}_{2}$ change. Although $\mathrm{PaCO}_{2}$ is more precise representation of systemic $\mathrm{CO}_{2}$ concentration, it can only be obtained by intermittent arterial sampling, which makes it difficult to monitor continuously throughout general anesthesia. $\mathrm{ETCO}_{2}$ is routinely monitored during general anesthesia and it shows a good correlation with $\mathrm{PaCO}_{2}$, even in those with severe brain trauma [25,26]. Moreover, ICP variation has been reported to be associated with $\mathrm{ETCO}_{2}$ change [27]. Therefore, $\mathrm{ETCO}_{2}$ may be a more convenient and appropriate method to evaluate dynamic changes in ONSD than that of monitoring $\mathrm{PaCO}_{2}$.

This study had several limitations. First, there was no gold standard for ICP measurement. Although direct ICP measurement technique such as ventriculostomy is known as a standard, it is invasive and difficult to apply as routine monitoring of all patients likely to experience increased ICP, even in critically ill patients. Sonographic measurement of ONSD is a non-invasive technique and has been reported to provide a good association with ICP $[21,28,29]$. Thus, ONSD can be a good alternative in predicting ICP increase without risk of infection or bleeding. A second limitation is that of $\mathrm{ETCO}_{2}$, which is a non-invasive estimate of $\mathrm{PaCO}_{2}$ that might be affected by other factors such as decreased cardiac output [30]. Although $\mathrm{ETCO}_{2}$ was compared with ONSD in the present study and showed a good correlation, $\mathrm{PaCO}_{2}$ can reflect systemic $\mathrm{CO}_{2}$ level more accurately. The differences between $\mathrm{PaCO}_{2}$ and $\mathrm{ETCO}_{2}$ in the initial ventilator setting of individual patients should be calibrated for more reliability. However, demographic data in the present study showed no differences in hemodynamic parameters and the differences between $\mathrm{ETCO}_{2}$ and $\mathrm{PaCO}_{2}$ were not significantly changed between the two $\mathrm{ETCO}_{2}$ levels indicating that $\mathrm{ETCO}_{2}$ was minimally influenced by other confounding factors. Third, the accuracy of the ONSD measurement through PACS by the second observer might raise an issue. Because the second observer calculated ONSD by measuring the numbers of pixels as oppose to using the caliper provided by the ultrasound machine directly. Considering this discrepancy, we tested the accuracy of measuring ONSD through PACS prior to the study. The ONSD measured through PACS were very close to the measured by the ultrasound machine's caliper. Although the values between directly measured ONSD and the ONSD measured through PACS may not be valid for inter-observer variability, the consistency of the sonographic plane is preferable to the continuous ONSD measurement. Thus, despite of its limits, using saved image for second ONSD measurement was inevitable and the interobserver variability of the present study showed similar results to the previous studies [22].

In conclusion, ONSD may reflect the dynamic changes in ICP associated with arterial $\mathrm{CO}_{2}$ change during general anesthesia, which lends to support that monitoring $\mathrm{ETCO}_{2}$ is important in neurocritically-ill patients. In patients with the risk of ICP increase, ONSD may be used as a quick and easy screening tool for detection of increased ICP and also be useful to evaluate ICP sequentially without any invasive procedure at bedside during mechanical ventilation.

\section{References}

1. Petersen KD, Landsfeldt U, Cold GE, Petersen CB, Mau S, Hauerberg J, et al. Intracranial pressure and cerebral hemodynamic in patients with cerebral tumors: a randomized prospective study of patients subjected to craniotomy in propofol-fentanyl, isoflurane-fentanyl, or sevoflurane-fentanyl anesthesia. Anesthesiology 2003; 98: 329-36.

2. Nishiyama T, Matsukawa T, Yokoyama T, Hanaoka K. Cerebrovascular carbon dioxide reactivity during general anesthesia: a comparison between sevoflurane and isoflurane. Anesth Analg 1999; 89: 1437-41.

3. Vigue B, Ract C, Zlotine N, Leblanc PE, Samii K, Bissonnette B. Relationship between intracranial pressure, mild hypothermia and temperature-corrected $\mathrm{PaCO} 2$ in patients with traumatic brain injury. Intensive Care Med 2000; 26: 722-8.

4. Go SL, Singh JM. Pro/con debate: should PaCO2 be tightly controlled in all patients with acute brain injuries? Crit Care 2013; 17: 202.

5. Stiefel MF, Spiotta A, Gracias VH, Garuffe AM, Guillamondegui O, Maloney-Wilensky E, et al. Reduced mortality rate in patients with severe traumatic brain injury treated with brain tissue oxygen monitoring. J Neurosurg 2005; 103: 805-11.

6. Isert P. Control of carbon dioxide levels during neuroanaesthesia: current practice and an appraisal of our reliance upon capnography. Anaesth Intensive Care 1994; 22: 435-41. 
7. Russell GB, Graybeal JM. End-tidal carbon dioxide as an indicator of arterial carbon dioxide in neurointensive care patients. J Neurosurg Anesthesiol 1992; 4: 245-9.

8. Olivecrona M, Rodling-Wahlstrom M, Naredi S, Koskinen LO. Effective ICP reduction by decompressive craniectomy in patients with severe traumatic brain injury treated by an ICP-targeted therapy. J Neurotrauma 2007; 24: 927-35.

9. Geeraerts T, Merceron S, Benhamou D, Vigue B, Duranteau J. Non-invasive assessment of intracranial pressure using ocular sonography in neurocritical care patients. Intensive Care Med 2008; 34: 2062-7.

10. Arabi Y, Memish ZA, Balkhy HH, Francis C, Ferayan A, Al Shimemeri A, et al. Ventriculostomy-associated infections: incidence and risk factors. Am J Infect Control 2005; 33: 137-43.

11. Soldatos T, Karakitsos D, Chatzimichail K, Papathanasiou M, Gouliamos A, Karabinis A. Optic nerve sonography in the diagnostic evaluation of adult brain injury. Crit Care 2008; 12: R67.

12. Hansen HC, Lagreze W, Krueger O, Helmke K. Dependence of the optic nerve sheath diameter on acutely applied subarachnoidal pressure - an experimental ultrasound study. Acta Ophthalmol 2011; 89: e528-32.

13. Rajajee V, Fletcher JJ, Rochlen LR, Jacobs TL. Comparison of accuracy of optic nerve ultrasound for the detection of intracranial hypertension in the setting of acutely fluctuating vs stable intracranial pressure: post-hoc analysis of data from a prospective, blinded single center study. Crit Care 2012; 16: R79.

14. Seo H, Kim YK, Shin WJ, Hwang GS. Ultrasonographic optic nerve sheath diameter is correlated with arterial carbon dioxide concentration during reperfusion in liver transplant recipients. Transplant Proc 2013; 45: 2272-6.

15. Smith M. Monitoring intracranial pressure in traumatic brain injury. Anesth Analg 2008; 106: 240-8.

16. Marshall LF, Smith RW, Shapiro HM. The outcome with aggressive treatment in severe head injuries. Part II: acute and chronic barbiturate administration in the management of head injury. J Neurosurg 1979; 50: 26-30.

17. Bacani CJ, Freeman WD, Di Trapani RA, Canabal JC, Arasi L, Shine T, et al. Emergent, controlled lumbar drainage for intracranial pressure monitoring during orthotopic liver transplantation. Neurocrit Care 2011; 14: 447-52.

18. Lidofsky SD, Bass NM, Prager MC, Washington DE, Read AE, Wright TL, et al. Intracranial pressure monitoring and liver transplantation for fulminant hepatic failure. Hepatology 1992; 16: 1-7.

19. Steiner LA, Balestreri M, Johnston AJ, Czosnyka M, Coles JP, Chatfield DA, et al. Sustained moderate reductions in arterial CO2 after brain trauma time-course of cerebral blood flow velocity and intracranial pressure. Intensive Care Med 2004; 30: 2180-7.

20. Newell DW, Weber JP, Watson R, Aaslid R, Winn HR. Effect of transient moderate hyperventilation on dynamic cerebral autoregulation after severe head injury. Neurosurgery 1996; 39: 35-43.

21. Kristiansson H, Nissborg E, Bartek J Jr, Andresen M, Reinstrup P, Romner B. Measuring elevated intracranial pressure through noninvasive methods: a review of the literature. J Neurosurg Anesthesiol 2013; 25: 372-85.

22. Moretti R, Pizzi B. Ultrasonography of the optic nerve in neurocritically ill patients. Acta Anaesthesiol Scand 2011; 55: 644-52.

23. Ballantyne SA, O'Neill G, Hamilton R, Hollman AS. Observer variation in the sonographic measurement of optic nerve sheath diameter in normal adults. Eur J Ultrasound 2002; 15: 145-9.

24. Helmke K, Hansen HC. Fundamentals of transorbital sonographic evaluation of optic nerve sheath expansion under intracranial hypertension II. Patient study. Pediatr Radiol 1996; 26: 706-10.

25. Kerr ME, Zempsky J, Sereika S, Orndoff P, Rudy EB. Relationship between arterial carbon dioxide and end-tidal carbon dioxide in mechanically ventilated adults with severe head trauma. Crit Care Med 1996; 24: 785-90.

26. Cinar O, Acar YA, Arziman I, Kilic E, Eyi YE, Ocal R. Can mainstream end-tidal carbon dioxide measurement accurately predict the arterial carbon dioxide level of patients with acute dyspnea in ED. Am J Emerg Med 2012; 30: 358-61.

27. Kim S, McNames J, Goldstein B. Intracranial pressure variation associated with changes in end-tidal CO2. Conf Proc IEEE Eng Med Biol Soc 2006; 1: 9-12.

28. Strumwasser A, Kwan RO, Yeung L, Miraflor E, Ereso A, Castro-Moure F, et al. Sonographic optic nerve sheath diameter as an estimate of intracranial pressure in adult trauma. J Surg Res 2011; 170: 265-71.

29. Tayal VS, Neulander M, Norton HJ, Foster T, Saunders T, Blaivas M. Emergency department sonographic measurement of optic nerve sheath diameter to detect findings of increased intracranial pressure in adult head injury patients. Ann Emerg Med 2007; 49: 508-14.

30. Shibutani K, Muraoka M, Shirasaki S, Kubal K, Sanchala VT, Gupte P. Do changes in end-tidal PCO2 quantitatively reflect changes in cardiac output? Anesth Analg 1994; 79: 829-33. 